\title{
The Coronal Isotopic Composition as Determined Using Solar Energetic Particles
}

\author{
R. A. Leske*, R. A. Mewaldt*, C. M. S. Cohen*, E. R. Christian ${ }^{\dagger}$, A. C. Cummings*, \\ P. L. Slocum ${ }^{* *}$, E. C. Stone*, T. T. von Rosenvinge ${ }^{\dagger}$ and M. E. Wiedenbeck ${ }^{* *}$ \\ ${ }^{*}$ California Institute of Technology, Pasadena, CA 91125 USA \\ ${ }^{\dagger}$ NASA/Goddard Space Flight Center, Greenbelt, MD 20771 USA \\ ** Jet Propulsion Laboratory, Pasadena, CA 91109 USA \\ $\ddagger$ Presently at The Aerospace Corporation, El Segundo, CA 90245 USA
}

\begin{abstract}
Solar energetic particles (SEPs), like the solar wind, provide a direct sample of the Sun. Although SEP abundances show a variable amount of mass fractionation, it is possible to develop methods of correcting for it in order to deduce the composition of the corona. Using high-resolution measurements from the Solar Isotope Spectrometer on the Advanced Composition Explorer, we have studied the isotopic composition of 10 abundant elements from C to Ni in 32 large SEP events from late 1997 to the end of 2001 at energies > $15 \mathrm{MeV}$ /nucleon. We show that various isotopic and elemental enhancements are correlated with each other, discuss the first order corrections used to account for the variability, and obtain estimated coronal abundances. We compare the coronal values and their uncertainties inferred from SEPs with those that are available from solar wind and meteoritic measurements and find generally good agreement. We include $\mathrm{C}$ and Ni isotopic abundances, for which no solar wind measurements have yet been reported.
\end{abstract}

\section{INTRODUCTION}

Studies of solar material in both the solar wind and solar energetic particles (SEPs) may be used to determine the Sun's composition, and each approach has its own challenges. For example, solar wind composition measurements typically require detailed knowledge of instrument response functions, effi ciencies, and backgrounds, whereas SEP abundances are often highly skewed by fractionation processes during particle acceleration or transport. It is therefore valuable to compare solar abundances obtained from both types of studies.

The seed material for gradual SEP events is thought to be solar wind or coronal material accelerated by large shocks driven by fast coronal mass ejections [1]. Elemental abundances vary greatly from event to event but are correlated with the ionic charge to mass ratio, $Q / M$ [2]. After correcting for this fractionation [2,3] or averaging over many events [4], SEP abundances reveal the coronal elemental composition. In principle, the coronal isotopic composition can be similarly determined [5, 6].

Recent studies using the Solar Isotope Spectrometer (SIS) on the Advanced Composition Explorer (ACE) have found large enhancements and event-to-event variability in SEP isotopic abundance ratios [see, e.g. 7, and references therein]. In the present work, we present ACE/SIS isotopic abundance measurements for $\mathrm{C}, \mathrm{O}$,
$\mathrm{Ne}, \mathrm{Mg}, \mathrm{Si}, \mathrm{S}, \mathrm{Ar}, \mathrm{Ca}, \mathrm{Fe}$, and $\mathrm{Ni}$ in as many as 32 individual SEP events. Although the isotopic composition is highly variable, we can use the observed correlations to empirically correct for the variations to fi rst order and obtain coronal isotopic abundances from SEPs, which we compare with solar wind and terrestrial abundances.

\section{OBSERVATIONS AND ANALYSIS}

The SIS instrument uses the $\mathrm{d} E / \mathrm{d} x$ versus residual energy technique in a pair of silicon solid-state detector telescopes to obtain the nuclear charge, $Z$, mass, $M$, and total kinetic energy, $E$, for particles with energies of $\sim 10$ to $\sim 100 \mathrm{MeV} /$ nucleon [8]. For this study, we examined all SEP events with high-energy heavy ion fluxes large enough to yield statistically meaningful isotope abundances for at least a few isotope ratios. This selection resulted in 32 large SEP events, including the 18 discussed in our earlier work [7] and an additional 14 in 2001. During the very highest rate periods, mass resolution is degraded by chance coincidences between heavy ions and low energy protons. Therefore, time periods near the peaks of the 14 July 2000, 9 November 2000, 24 September 2001, 4 November 2001, and 22 November 2001 events were not used for the isotopic analysis. Mass resolution depends on $Z$ and $E$; for the species 
and energies studied here it ranges from $\sim 0.15$ to $\sim 0.3$ amu. Obtaining event-integrated isotope abundance ratios from these high-resolution data is straightforward; further details are given elsewhere $[9,10]$.

Deriving coronal abundances from SEP data is complicated by the fact that SEP isotopic abundances may vary signifi cantly from event to event [10], as shown for the ${ }^{22} \mathrm{Ne} /{ }^{20} \mathrm{Ne}$ ratio in Figure 1. There is some indication that this variability may depend on the phase of the solar cycle [11]; further investigation may help determine if this is indeed the case or if the apparent changes in variability are merely statistical aberrations.

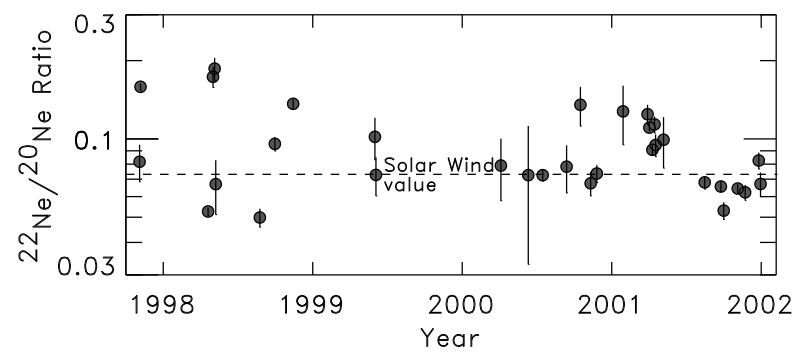

FIGURE 1. The $\mathrm{SEP}{ }^{22} \mathrm{Ne} /{ }^{20} \mathrm{Ne}$ ratio measured by ACE/SIS at $E>15 \mathrm{MeV} /$ nucleon plotted versus the date of the event, compared with the average solar wind value [12] (dashed line).

As mentioned above, earlier studies [e.g. 2] have shown that SEP heavy ion elemental abundances are also quite variable from event to event, with abundance ratios that scale approximately as a power law in $Q / M$ with a different power law index for each SEP event. Any process which fractionates elements based on $Q / M$ will also affect isotopes with different $M$, and there should be a predictable correlation between elemental and isotopic abundances. Following [5], if we base the power law fractionation index on the abundance ratio of any two reference species, such as $\mathrm{Fe} / \mathrm{O}, \mathrm{Na} / \mathrm{Mg}$, or, in general terms, $\mathrm{R}_{1} / \mathrm{R}_{2}$, power-law fractionation in $Q / M$ implies that the enhancement or depletion of any other SEP abundance ratio $\mathrm{X}_{1} / \mathrm{X}_{2}$ should be:

$\frac{\left(\mathrm{X}_{1} / \mathrm{X}_{2}\right)_{\mathrm{SEP}}}{\left(\mathrm{X}_{1} / \mathrm{X}_{2}\right)_{\text {corona }}}=\left(\frac{\left(\mathrm{R}_{1} / \mathrm{R}_{2}\right)_{\mathrm{SEP}}}{\left(\mathrm{R}_{1} / \mathrm{R}_{2}\right)_{\text {corona }}}\right)^{\frac{\ln \left[(Q / M) \mathrm{X}_{1} /(Q / M)_{\mathrm{X}}\right]}{\ln \left[(Q / M)_{\mathrm{R}_{1} /(Q / M)_{2}}\right]}}$.

We fi nd that isotopic abundances are indeed correlated with elemental abundances, as illustrated in Figure 2, which reinforces the trends seen with many fewer events in earlier work [10]. To compare the correlations with those expected from equation (1), the ionic charge states $Q$ of the species involved must be known. It is reasonable to assume that $Q\left({ }^{22} \mathrm{Ne}\right)=Q\left({ }^{20} \mathrm{Ne}\right)$, so the values of $Q\left(\mathrm{X}_{1}\right)$ and $Q\left(\mathrm{X}_{2}\right)$ in equation (1) factor out, but this will not be true for the charge states of $\mathrm{Fe} / \mathrm{O}$ or $\mathrm{Na} / \mathrm{Mg}$ used in Figure 2. For most SEP events $Q$ is not measured at energies of tens of $\mathrm{MeV} /$ nucleon. Lower energy measurements may not apply as several events have been found

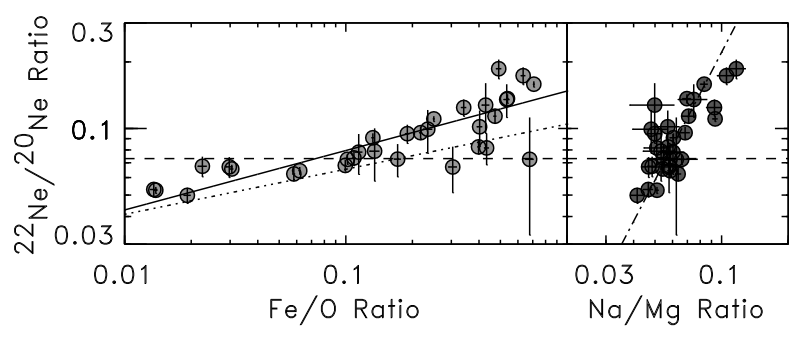

FIGURE 2. The SEP ${ }^{22} \mathrm{Ne} /{ }^{20} \mathrm{Ne}$ isotopic ratio plotted versus the $\mathrm{Fe} / \mathrm{O}$ (left) and $\mathrm{Na} / \mathrm{Mg}$ (right) elemental abundance ratios. Diagonal lines show the correlations expected from equation (1), assuming parameters discussed in the text. The horizontal dashed line marks the solar wind ${ }^{22} \mathrm{Ne} /{ }^{20} \mathrm{Ne}$ value.

with energy-dependent charge states for heavy elements [e.g. 13], while those higher energy charge state measurements that do exist show considerable event-to-event variability [see, e.g. 14, and references therein]. Also, note that in the derivation of equation (1) it was implicitly assumed that any elemental fractionation associated with the fi rst ionization potential (FIP) effect is the same magnitude in the SEP event as it is in the corona. However, the size of the FIP effect in SEP events is variable $[15,16]$, which can affect certain elemental but not isotopic ratios and may blur the expected correlations.

If we evaluate equation (1) with $Q(\mathrm{Fe})=15$ [17] (and a FIP step of 4), we obtain the dotted line in Figure 2. If instead we use $Q(\mathrm{Fe})=18$ and a reduced FIP step of 1.7, both of which were found for the 6 November 1997 event [18], we obtain the steeper solid line. Mean iron charge states both lower than 15 and higher than 18 have been observed [14], so the illustrated lines indicate only some of the range of variability that might be expected.

The ${ }^{22} \mathrm{Ne} /{ }^{20} \mathrm{Ne}$ ratio correlates much better with $\mathrm{Na} / \mathrm{Mg}$, as shown in the right panel of Figure 2. Note that those events with ${ }^{22} \mathrm{Ne} /{ }^{20} \mathrm{Ne}$ values near that of the solar wind exhibit $\mathrm{Fe} / \mathrm{O}$ ratios which span nearly 2 orders of magnitude, while all their $\mathrm{Na} / \mathrm{Mg}$ ratios are tightly clustered around the coronal value. $\mathrm{Na}$ and $\mathrm{Mg}$ have similar FIP values, and their charge states are much less variable than those of Fe. As noted in [19], both $\mathrm{Na}$ and $\mathrm{Mg}$ ions should have $\sim 2$ electrons attached over a broad range of coronal temperatures [20], and ${ }^{23} \mathrm{Na}$ is neutron-rich compared to ${ }^{24} \mathrm{Mg}$, so they differ signifi cantly in $Q / M$. The dot-dashed line in Figure 2 shows the expected correlation if $Q(\mathrm{Na})=9$ and $Q(\mathrm{Mg})=10$. This very simple model provides a good fi rst order fit to the data, but the actual correlation line is a bit shallower than predicted.

The predicted correlations are very sensitive to $Q$ for reference ratios involving similar values of $Q / M$, and changing $Q(\mathrm{Na}) / Q(\mathrm{Mg})$ by only $1 \%$ changes the expected slope considerably [7]. Even if $Q$ could be measured to this accuracy at SIS energies, it is $Q$ at the time 


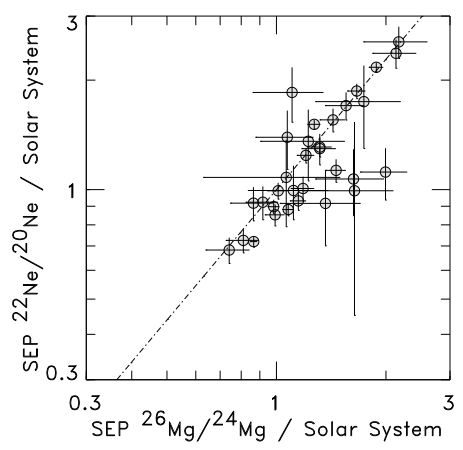

FIGURE 3. The ${ }^{22} \mathrm{Ne} /{ }^{20} \mathrm{Ne}$ versus ${ }^{26} \mathrm{Mg} /{ }^{24} \mathrm{Mg}$ isotopic ratios in each of the 32 SEP events, normalized to standard solar system values [21]. The diagonal line shows the correlation expected using equation (1).

of fractionation that is relevant, which may differ from that at $1 \mathrm{AU}$ if fractionation happens early and if stripping occurs in acceleration through the corona [see, e.g. 22]. The sensitivity of the correlations to variability in the $\mathrm{Na}$ and $\mathrm{Mg}$ charge states may account for the scatter still present in the right panel of Figure 2.

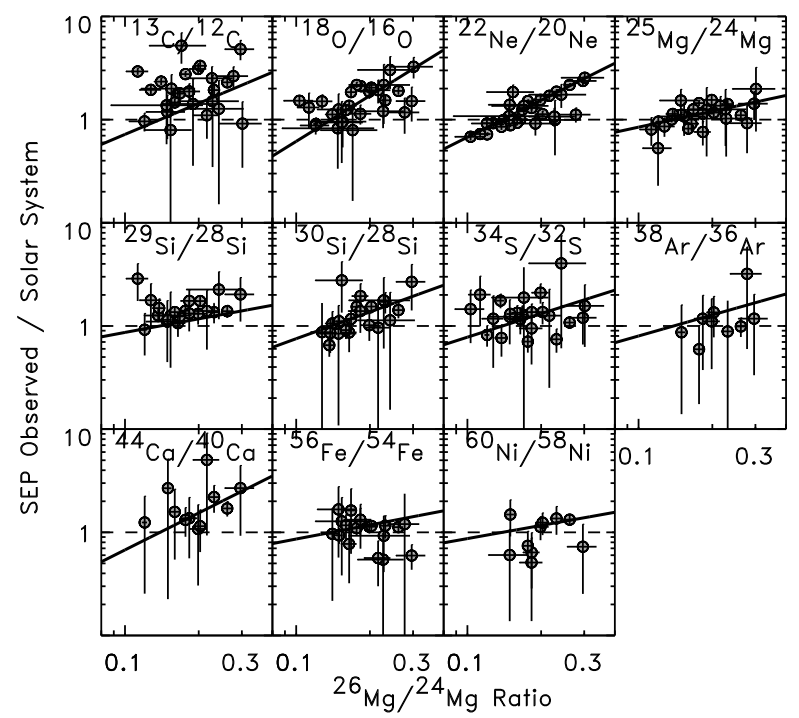

FIGURE 4. Eleven SEP isotope abundance ratios (normalized to standard solar system abundances [21]) in up to 32 SEP events plotted versus the ${ }^{26} \mathrm{Mg} /{ }^{24} \mathrm{Mg}$ ratio. Diagonal lines show the correlations expected using equation (1).

Since $Q$ should be the same for isotopes of the same element, if we use an isotope ratio as the reference value in equation (1) the charge states factor out and we might expect a tighter correlation. This is illustrated by the plot of ${ }^{22} \mathrm{Ne} /{ }^{20} \mathrm{Ne}$ versus ${ }^{26} \mathrm{Mg} /{ }^{24} \mathrm{Mg}$ in Figure 3 . Most of the events with small uncertainties agree very well with the expected line, while the outliers tend to have the largest uncertainties. Across the range of observed values no systematic deviation from the expected trend is evident.

\section{RESULTS}

The SEP abundance values for 11 isotope ratios for elements from $\mathrm{C}$ to $\mathrm{Ni}$ are shown plotted versus ${ }^{26} \mathrm{Mg} /{ }^{24} \mathrm{Mg}$ in Figure 4, with the correlations expected from equation (1) indicated by diagonal lines. The data roughly agree with the expected trends for $\mathrm{O}, \mathrm{Ne}, \mathrm{Mg}$, and $\mathrm{Si}$. Elements heavier than Si tend to have fewer data points and larger uncertainties, and it is unclear whether there is any fractionation at all, much less whether it follows the expected trends. The correlations may break down for species with $Q / M$ far from that of the $\mathrm{Mg}$ reference ratio if the actual dependence on $Q / M$ is not a simple power law as we assumed. At lower $Z,{ }^{13} \mathrm{C}$ is often enhanced or ${ }^{12} \mathrm{C}$ is depleted in SEP events relative to terrestrial abundances, but the reason for this is not understood. Higher ${ }^{13} \mathrm{C}$ values do not appear to be due to spillover from ${ }^{12} \mathrm{C}$, as the two mass peaks are generally well separated [9].

Using ${ }^{26} \mathrm{Mg} /{ }^{24} \mathrm{Mg}$ as the reference ratio, taking its coronal value to be the terrestrial value [21], and assuming $Q$ is the same for different isotopes of the same element, we solve equation (1) for the coronal isotope ratios for each SEP event. Averaging over all the SIS measurements for each isotope ratio results in the SEPderived coronal values shown in Figure 5. We also show the weighted average without correcting for fractionation, which may be more appropriate for cases where the data may not follow the expected fractionation correlations. Also, the uncorrected number gives the average SEP composition arriving at $1 \mathrm{AU}$, and for ${ }^{22} \mathrm{Ne} /{ }^{20} \mathrm{Ne}$ we find it to be consistent with the value of $\sim 0.09$ of the so-called SEP component found implanted in lunar soils [23] (but see also [24]). Our SEP-derived values are also compared with standard solar system values [21] and existing solar wind values [12, 25, 26, 27] in Figure 5.

Although our fractionation correction is simplistic, the resulting coronal abundances mostly appear to be quite reasonable. All but 2 values are within $2 \sigma$ of the standard "solar system" values [21] (which are actually terrestrial values except for $\mathrm{Ne}$ and $\mathrm{Ar}$, for which solar wind values were used). Neither $\mathrm{C}$ nor $\mathrm{Ni}$ isotope abundances have yet been reported from solar wind data. Our ${ }^{13} \mathrm{C}$ measurements suggest signifi cant additional fractionation of SEP C isotopes, but ${ }^{60} \mathrm{Ni} /{ }^{58} \mathrm{Ni}$ appears to be much less variable and the average is more likely representative of the corona. For ratios such as ${ }^{18} \mathrm{O} /{ }^{16} \mathrm{O},{ }^{34} \mathrm{~S} /{ }^{32} \mathrm{~S}$, and ${ }^{54} \mathrm{Fe} /{ }^{56} \mathrm{Fe}$, uncertainties on the SEP-derived coronal abundances are comparable to those obtained from the solar wind. Additional SEP events may reduce the uncertainties for the heaviest species where there are still only a few measurements, but a better theoretical understanding of the mass fractionation process would allow much further progress. Continued study of puzzles such as apparent changes in fractionation with the solar cycle and the frequent enhancement of ${ }^{13} \mathrm{C}$ may provide clues 


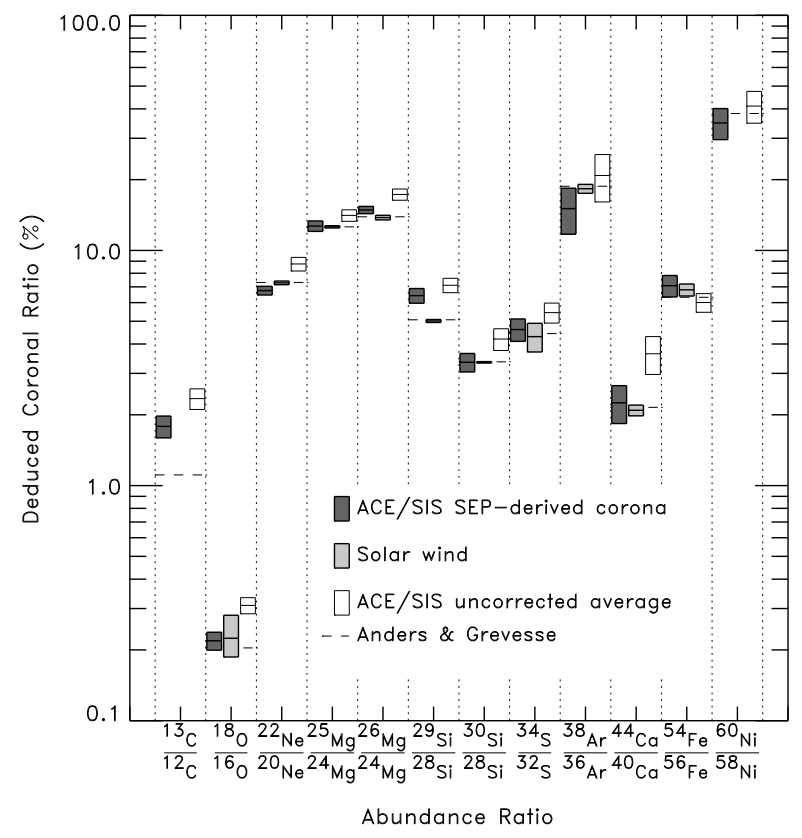

FIGURE 5. Average coronal isotopic abundance ratios from SIS SEP measurements after correcting for fractionation (dark grey boxes). The ${ }^{26} \mathrm{Mg} /{ }^{24} \mathrm{Mg}$ ratio served as the reference value in equation (1) for everything other than the ${ }^{26} \mathrm{Mg} /{ }^{24} \mathrm{Mg}$ ratio, for which ${ }^{22} \mathrm{Ne} /{ }^{20} \mathrm{Ne}$ was used. For comparison, averages without fractionation corrections (open boxes), standard solar system values (dashed lines; [21]) and measured solar wind values (light grey boxes; [12, 25, 26, 27]) are shown.

to the nature of the fractionation process.

\section{ACKNOWLEDGMENTS}

This work was supported by NASA at the California Institute of Technology (grant NAG5-6912), the Jet Propulsion Laboratory, and the Goddard Space Flight Center.

\section{REFERENCES}

1. Reames, D. V., Revs. Geophys., 33, 585-589 (1995).

2. Breneman, H. H., and Stone, E. C., Astrophys. J. Lett., 299, L57-L61 (1985).

3. Garrard, T. L., and Stone, E. C., Proc. 23rd Internat. Cosmic Ray Conf. (Calgary), 3, 384-387 (1993).

4. Reames, D. V., Adv. Space Res., 15, (7)41-(7)51 (1995).

5. Mewaldt, R. A., and Stone, E. C., Astrophys. J., 337, 959-963 (1989).

6. Williams, D. L., Leske, R. A., Mewaldt, R. A., and Stone, E. C., Space Sci. Rev., 85, 379-386 (1998).

7. Leske, R. A., et al., "Isotopic Abundances in the Solar Corona as Inferred from ACE Measurements of Solar Energetic Particles," in Solar and Galactic Composition, edited by R. F. Wimmer-Schweingruber, AIP Conf. Proc. 598, AIP, New York, 2001, pp. 127-132.

8. Stone, E. C., et al., Space Sci. Rev., 86, 357-408 (1998).

9. Leske, R. A., et al., Geophys. Res. Lett., 26, 153-156 (1999).

10. Leske, R. A., et al., Geophys. Res. Lett., 26, 2693-2696 (1999).

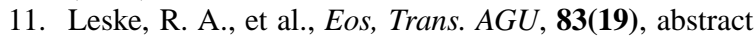
SH42B-04 (2002).

12. Kallenbach, R., "Isotopic Composition Measured In-Situ in Different Solar Wind Regimes by CELIAS/MTOF on Board SOHO," in Solar and Galactic Composition, edited by R. F. Wimmer-Schweingruber, AIP Conf. Proc. 598, AIP, New York, 2001, pp. 113-119.

13. Mazur, J. E., Mason, G. M., Looper, M. D., Leske, R. A., and Mewaldt, R. A., Geophys. Res. Lett., 26, 173-176 (1999).

14. Leske, R. A., et al., "The Ionic Charge State Composition at High Energies in Large Solar Energetic Particle Events in Solar Cycle 23," in Solar and Galactic Composition, edited by R. F. Wimmer-Schweingruber, AIP Conf. Proc. 598, AIP, New York, 2001, pp. 171-176.

15. Garrard, T. L., and Stone, E. C., Adv. Space Res., 14, (10)589-(10)598 (1994).

16. Mewaldt, R. A., et al., "Variable Fractionation of Solar Energetic Particles According to First Ionization Potential," in Acceleration and Transport of Energetic Particles Observed in the Heliosphere, edited by R. A. Mewaldt et al., AIP Conf. Proc. 528, AIP, New York, 2000, pp. 123-126.

17. Luhn, A., et al., Proc. 19th Internat. Cosmic Ray Conf. (La Jolla), 4, 241-244 (1985).

18. Cohen, C. M. S., et al., Geophys. Res. Lett., 26, 149-152 (1999).

19. Cohen, C. M. S., et al., Geophys. Res. Lett., 26, 2697-2700 (1999).

20. Arnaud, M., and Rothenflug, R., Astron. Astrophys. Suppl., 60, 425-457 (1985).

21. Anders, E., and Grevesse, N., Geochim. Cosmochim. Acta, 53, 197-214 (1989).

22. Barghouty, A. F., and Mewaldt, R. A., "Simulation of Charge-Equilibration and Acceleration of Solar Energetic Ions," in Acceleration and Transport of Energetic Particles Observed in the Heliosphere, edited by R. A. Mewaldt et al., AIP Conf. Proc. 528, AIP, New York, 2000, pp. 71-78.

23. Wieler, R., Space Sci. Rev., 85, 303-314 (1998).

24. Mewaldt, R. A., Ogliore, R. C., Gloeckler, G., and Mason, G. M., "A New Look at Neon-C and SEP-Neon," in Solar and Galactic Composition, edited by R. F. WimmerSchweingruber, AIP Conf. Proc. 598, AIP, New York, 2001, pp. 393-398.

25. Wimmer-Schweingruber, R. F., Bochsler, P., and Gloeckler, G., Geophys. Res. Lett., 28, 2763-2766 (2001).

26. Ipavich, F. M., Paquette, J. A., Bochsler, P., Lasley, S. E., and Wurz, P., "Solar Wind Iron Isotopic Abundances: Results from SOHO/CELIAS/MTOF," in Solar and Galactic Composition, edited by R. F. WimmerSchweingruber, AIP Conf. Proc. 598, AIP, New York, 2001, pp. 121-126.

27. Wimmer-Schweingruber, R. F., Adv. Space Res. (2002), in press. 